\section{Violência intrafamiliar contra crianças e adolescentes: identificação, manejo e conhecimento da rede de referência por fonoaudiólogo em serviços públicos de saúde}

\section{Family violence against children and adolescents: identification, management and knowledge of domestic violence against children and adolescents at public phonoaudiology referral services}

Raquel Moura Lins Acioli 1 Maria Luiza Carvalho de Lima 2

Maria Cynthia Braga 3

Fernando Castim Pimentel 4 Adriana Guerra de Castro 5
1 Programa Integrado de Pós-graduação em Saúde Coletiva. Universidade Federal de Pernambuco. Av. Prof Moraes Rego, s.n. Hospital das Clínicas. Bloco E, $4^{\circ}$ andar. Cidade Universitária. Recife, PE, Brasil. CEP: 50.670-901.

E-mail: raquelmlacioli@hotmail.com

2-4 Centro de Pesquisas Aggeu Magalhães. Fundação Oswaldo Cruz. Recife, PE, Brasil.

5 Programa de Pós-graduação Saúde da Criança e do Adolescente. Universidade Federal de Pernambuco. Recife, PE, Brasil.

\begin{abstract}
Objectives: to describe the profile and the conduct of public sector, philanthropic, and private phonoaudiologists contracted by the public health authorities in the city of Recife, in the Brazilian State of Pernambuco, with regard to suspected and/or confirmed cases of domestic violence against children and adolescents between August and October 2008.

Methods: a cross-sectional descriptive study was carried out with phonoaudiologists from the Brazilian public health service with more than one year of work experience following graduation. The study variables included socio-demographic characteristics, area of work, academic profile and suspected and/or confirmed cases of abuse reported by them and were obtained using a structured questionnaire, subsequently analyzed statistically using the chi-square and Fisher's Exact test.

Results: of the 89 phonoaudiologists, $43.8 \%$ (39) had attended cases of suspected and/or confirmed abuse, with physical abuse being the most frequent kind (35\%). Of the 70 cases reported, 2.9\% (2) were referred to the appropriate authorities. Most referred the cases to social services or psychologists (34.3\%). Around $50 \%$ of such patients abandoned treatment.

Conclusions: the reporting of cases was found to be very low, due, possibility, to lack of information on the part of phonoaudiologists as to the measures to be taken. This suggests that there is a need for professional training to improve the identification of cases of violence and teach phono-audiologists how to deal with them.
\end{abstract}

Key words Child, Adolescent, Speech, language and hearing sciences, Domestic violence

\section{Resumo}

Objetivos: descrever o perfil e a conduta dos fonoaudiólogos das redes pública, filantrópica e privada conveniada com o SUS da cidade do Recife, Pernambuco, diante dos casos suspeitos elou confirmados de violência contra crianças e adolescentes, de agosto a outubro de 2008

Métodos: estudo descritivo de corte transversal que envolveu os fonoaudiólogos da rede do SUS com mais de um ano de formação. Constituíram variáveis do estudo as características sociodemográficas, área de atuação profissional, informações sobre o perfil acadêmico dos profissionais e dos casos suspeitos elou confirmados de violência por eles relatados, obtidas a partir da utilização de questionário estruturado, analisados posteriormente através de frequências simples e sumarizadas pelo teste de quiquadrado e teste Exato de Fisher.

Resultados: dos 89 fonoaudiólogos, 43,8\% (39) atenderam casos suspeitos elou confirmados de violência, sendo a violência física a mais frequente (35\%). Dos 70 casos relatados, 2,9\% (2) foram denunciados aos órgãos competentes. A maioria encaminhou os casos para atendimento pelo serviço social ou psicológico (34,3\%). Cerca de $50 \%$ dos casos abandonou o tratamento.

Conclusões: verificou-se um reduzido número de notificações de violência ocasionado, possivelmente, pela pouca informação dos fonoaudiólogos sobre as medidas a serem tomadas, o que sugere a necessidade de capacitação profissional para uma melhor identificação e conduta frente aos casos de violência.

Palavras-chave Criança, Adolescente, Fonoaudiologia, Violência doméstica 


\section{Introdução}

Apesar do crescente conhecimento da violência no âmbito familiar e da obrigatoriedade da notificação estabelecida pelo Estatuto da Criança e do Adolescente (ECA), no Brasil, o sub-registro dos casos ainda é um problema. A atuação do profissional de saúde na identificação e prevenção da violência contra criança e adolescente é de fundamental importância, pois pode determinar o seu rompimento e impedir a (re)produção do ciclo da violência intrafamiliar. ${ }^{1}$

No Brasil, não existem dados epidemiológicos confiáveis sobre violência contra criança e adolescente, mas apenas registros isolados. Apesar da violência infantil ser frequente no país, estima-se que apenas $20 \%$ das ocorrências sejam denunciadas, sendo a notificação dos casos aos órgãos competentes uma pratica pouco exercida. 2

Estudos recentes têm sido desenvolvidos com o objetivo de diagnosticar a conduta, o preparo e a responsabilidade dos profissionais diante de casos de violência intrafamiliar no sistema de saúde.3-5 Seus resultados têm revelado o despreparo da maioria dos profissionais no enfrentamento do problema, principalmente ocasionado pela falta de acesso à informação, desde a graduação, pela ausência de treinamento específico e de apoio em seus diversos âmbitos de atuação. Essa situação agrava-se ainda mais pelo predomínio da atuação isolada dos profissionais, ocasionado pela pouca prática interdisciplinar, em que o manejo do problema é dirigido principalmente à conversa com a família, não seguida, na maioria das vezes, da notificação quando necessária.4,6

A identificação da violência não é fácil, devido a sua complexidade, além de depender de aspectos emocionais dos profissionais, estruturais, legais, da existência de órgãos de apoio e oferta de capacitações para a identificação dos sinais. No entanto, muitos profissionais, mesmo aqueles capacitados, temem a represália dos agressores, razão pela qual não notificam os casos de violência. 7,8

A dificuldade encontrada pelos profissionais a respeito da conduta correta a ser tomada diante de crianças e/ou adolescentes suspeitos e/ou confirmados de sofrerem violência é um dos fatores que proporciona a sua invisibilidade ao se defrontar com situações complexas de violência, isso porque, na sociedade, a prática do castigo e da autoridade é comum e reconhecida, frequentemente, como normal, principalmente no espaço considerado privado ou domiciliar, tida como forma de educar. ${ }^{2}$

$\mathrm{O}$ entendimento e a ação do fonoaudiólogo diante de casos de violência contra a criança e o adolescente são de extrema importância, especialmente em determinados segmentos da população infantil com maior risco de sofrer maus-tratos, como, por exemplo, os portadores de deficiências, anomalias congênitas e genéticas, que constituem grande percentual dos pacientes sob cuidados destes profissionais. 9,10

Estudos mostram que crianças com problemas de comunicação são mais vulneráveis ao abuso e/ou negligência decorrentes das dificuldades de comunicação, ${ }^{9,11}$ o que requer do fonoaudiólogo um olhar mais atento para a identificação dos casos de violência infanto-juvenil para, assim, poder adotar a melhor conduta e saber as origens reais das alterações da saúde geral do paciente. O fonoaudiólogo pode contribuir para a identificação desses casos, estando atento, por exemplo, às lesões ou cicatrizes na pele, particularmente aquelas localizadas na região da cabeça e pescoço, atingidos com maior frequência (cerca de 70\%).12,13

Observa-se uma escassez de trabalhos que retratem a conduta do fonoaudiólogo diante dos casos de violência intrafamiliar, o que difere de outras áreas no campo da saúde em que se observa um crescente número de publicações. 12,14-16 Tal fato é de fundamental importância, uma vez que esse profissional trabalha com crianças de risco, em sua maioria, por apresentarem distúrbios da comunicação humana, deficiências e problemas neurológicos. 4,6

Considerando a importância do tema, pela indisponibilidade de dados na região, o presente estudo teve como objetivo descrever o perfil dos fonoaudiólogos da cidade do Recife e a conduta deles diante dos casos suspeitos e/ou confirmados de violência contra crianças e adolescentes.

\section{Métodos}

O estudo de corte transversal, do tipo exploratório, incluiu o universo de fonoaudiólogos graduados há pelo menos um ano, que prestavam assistência à população infanto-juvenil usuária do Sistema Único de Saúde (SUS). A rede de assistência fonoaudiológica do município, no período de estudo, era composta por 22 serviços da rede pública e quatro de natureza filantrópica conveniada com o SUS. Excluíram-se os serviços privados não conveniados.

Após a obtenção da anuência pela Secretaria Municipal de Saúde do Recife e Secretaria Estadual de Saúde de Pernambuco, obteve-se uma lista dos serviços e informações sobre os profissionais e sua lotação. Dados relativos ao perfil profissional dos 
fonoaudiólogos e sua prática clínica frente aos casos suspeitos de violência, com ou sem confirmação, foram coletados no período de agosto a outubro de 2008.

A coleta de dados foi realizada utilizando o instrumento desenvolvido por Nouguchi e Assis, ${ }^{6}$ por apresentar questões referentes a áreas de atuação em fonoaudiologia e identificação dos tipos de violência (física, sexual, psicológica e negligência/ abandono), objeto do estudo. Porém, foram feitas algumas adaptações como inclusão de questões referentes à conduta do profissional frente aos casos de violência, nível acadêmico e participação de treinamento ou curso que abordasse o tema violência.

O estudo contemplou questões relativas ao reconhecimento, pelo profissional, de sinais de violência sofrida pela criança ou adolescente e sua conduta diante de tais situações. Além disso, foram aplicadas algumas questões formuladas por Deslandes, 17 com perguntas identificadoras de possíveis atendimentos a casos de violência, elaboradas a partir do instrumento da pesquisa "Prevenir e Proteger: Análise de Atenção à criança vítima de violência", utilizadas como uma forma de facilitar a identificação de possíveis casos de violência infantil nos serviços de saúde. Essas questões foram dirigidas aos profissionais que relataram não ter atendido casos suspeitos ou confirmados de violência durante o ano anterior à entrevista, e cuja resposta fornece pistas identificadoras da ocorrência de violência sofrida pela criança, referentes aos cuidados gerais (asseio corporal e atitudes disciplinares) e lesões corporais (queimadura, feridas, traumas). O questionário foi inicialmente testado em um estudo piloto com dez fonoaudiólogos.

As variáveis relativas à caracterização dos fonoaudiólogos foram sexo, tempo de formado, nível acadêmico, área de atuação e ter realizado ou não treinamento ou curso que tenha abordado o tema violência. As variáveis relativas aos pacientes atendidos e as condutas assumidas pelos fonoaudiólogos diante da suspeita de casos de violência foram: número de casos atendidos por profissional, tipo de violência suspeita ou confirmada, conduta tomada, continuidade do atendimento e conhecimento das instituições envolvidas na assistência à criança vitimizada. Foram analisadas informações relativas a casos atendidos no período de 2007 a 2008.

As informações foram digitadas e armazenadas em um banco de dados. Descreveu-se a distribuição de frequência das características relativas ao perfil do profissional e aos casos atendidos pelos profissionais. Não foi feita análise de comparabilidade entre os serviços públicos e privados conveniados com o SUS, levando em conta que são serviços igualmente públicos e em número reduzido. As diferenças na distribuição de frequência entre as variáveis categóricas foram testadas pelo teste de qui-quadrado de Pearson ou o Teste Exato de Fisherpara as situações cujas categorias apresentaram valores menores que cinco unidades. Os testes estatísticos foram considerados significantes quando o valor de $p<0,05$. O programa Epi-info, versão 3.3.2 for Windows, foi utilizado na entrada e análise dos dados.

O projeto foi aprovado pelo Comitê de Ética em Pesquisa em Seres Humanos do Instituto de Medicina Integral Prof. Fernando Figueira (IMIP) sob protocolo de $\mathrm{n}^{\circ} 1161$, conforme resolução 196/6. O questionário foi aplicado aos profissionais após a leitura e assinatura do termo de consentimento livre e esclarecido.

\section{Resultados}

Dos 92 fonoaudiólogos que prestavam assistência a usuários do SUS no período, 89 (96,7\%) participaram do estudo, dois estavam afastados por férias e um não foi localizado. Do total de participantes, 88 $(98,8 \%)$ eram do sexo feminino e 47 (52,8\%) haviam concluído o curso de graduação há mais de dez anos. Quanto às áreas de atuação, 40 (44,9\%) atuavam em mais de uma área e 19 (21,3\%) unicamente na área de audiologia e $68,5 \%$ (61) haviam cursado especialização e/ou residência. Entre os pós-graduados, 29 $(74,4 \%)$ declararam ter atendido a crianças e adolescentes vítimas de violência. Um total de dez profissionais $(11,2 \%)$ informou ter participado de cursos ou treinamento com algum tipo de abordagem em violência.

Entre os fonoaudiólogos estudados, 39 (43,8\%) informaram ter atendido a crianças ou adolescentes com quadros suspeitos ou confirmados de violência. Entre esses, $20(51,3 \%)$ atenderam a um caso, 13 $(33,3 \%)$ a dois casos, $2(5,1 \%)$ a três casos e 4 $(10,3 \%)$ atenderam quatro ou mais casos, totalizando 70 casos reportados. A violência física (35\%) e a negligência/abandono (26\%) foram os tipos de violência mais frequentemente referidos pelos profissionais (Figura 1). A maior proporção dos casos foi atendida por profissionais que atuavam em mais de uma área no campo da fonoaudiologia $(51,3 \%)$, seguida pelos generalistas $(20,5 \%)$, pelos profissionais que atuavam nas áreas de audiologia $(17,9 \%)$, motricidade orofacial $(5,1 \%)$, linguagem $(2,6 \%)$ e voz $(2,6 \%)$. Nenhuma característica do perfil profissional dos participantes do estudo esteve 
estatisticamente associada ao número de casos atendidos (Tabela 1).

Entre os $50(56,8 \%)$ profissionais que afirmaram nunca ter atendido casos suspeitos e/ou confirmados de violência, ao serem questionados sobre qual(is) situação(ões) reconheciam como caso de violência, $44(49,4 \%)$ responderam da seguinte forma: pai/mãe/responsável que grita com a criança ou adolescente, descrevendo-a como má ou desobediente $(n=27)$; deixar a criança a maior parte do tempo isolada $(n=39)$; desleixo com a higiene e aparência pessoal da criança $(n=44)$; crianças com vestimenta inadequada ao clima $(\mathrm{n}=29)$; problemas físicos ou necessidades não atendidas $(\mathrm{n}=40)$; pais que apresentam ter pouca atenção com a criança/ adolescente $(n=32)$; presença de lesões físicas, tais como queimaduras, feridas e fraturas discordantes das causas referidas $(n=44)$.
Dos 70 pacientes suspeitos ou confirmados de violência atendidos pelos fonoaudiólogos, houve relato de $34(48,6 \%)$ casos de abandono ao tratamento. As principais condutas tomadas pelos profissionais, diante dos casos de violência, foram: encaminhá-los ao serviço social ou psicológico ( 24 casos, $34,3 \%$ ), fornecer orientações à família de como proceder (18 casos, 25,7\%). Apenas 2,9\% das respostas referiram ter denunciado os casos aos órgãos competentes, quatorze profissionais não tiveram nenhuma conduta por ter recebido os pacientes encaminhados dos órgãos competentes. (Tabela 2). Entre as instituições responsáveis pela assistência à criança e ao adolescente vítima de violência, o Conselho Tutelar $(96,6 \%)$ foi a mais lembrada pelos profissionais entrevistados (Figura 2).

\section{Figura 1}

Distribuição de frequência dos tipos de violência suspeitos e/ou confirmados no atendimento fonoaudiológico na rede pública de saúde. Recife, 2008.

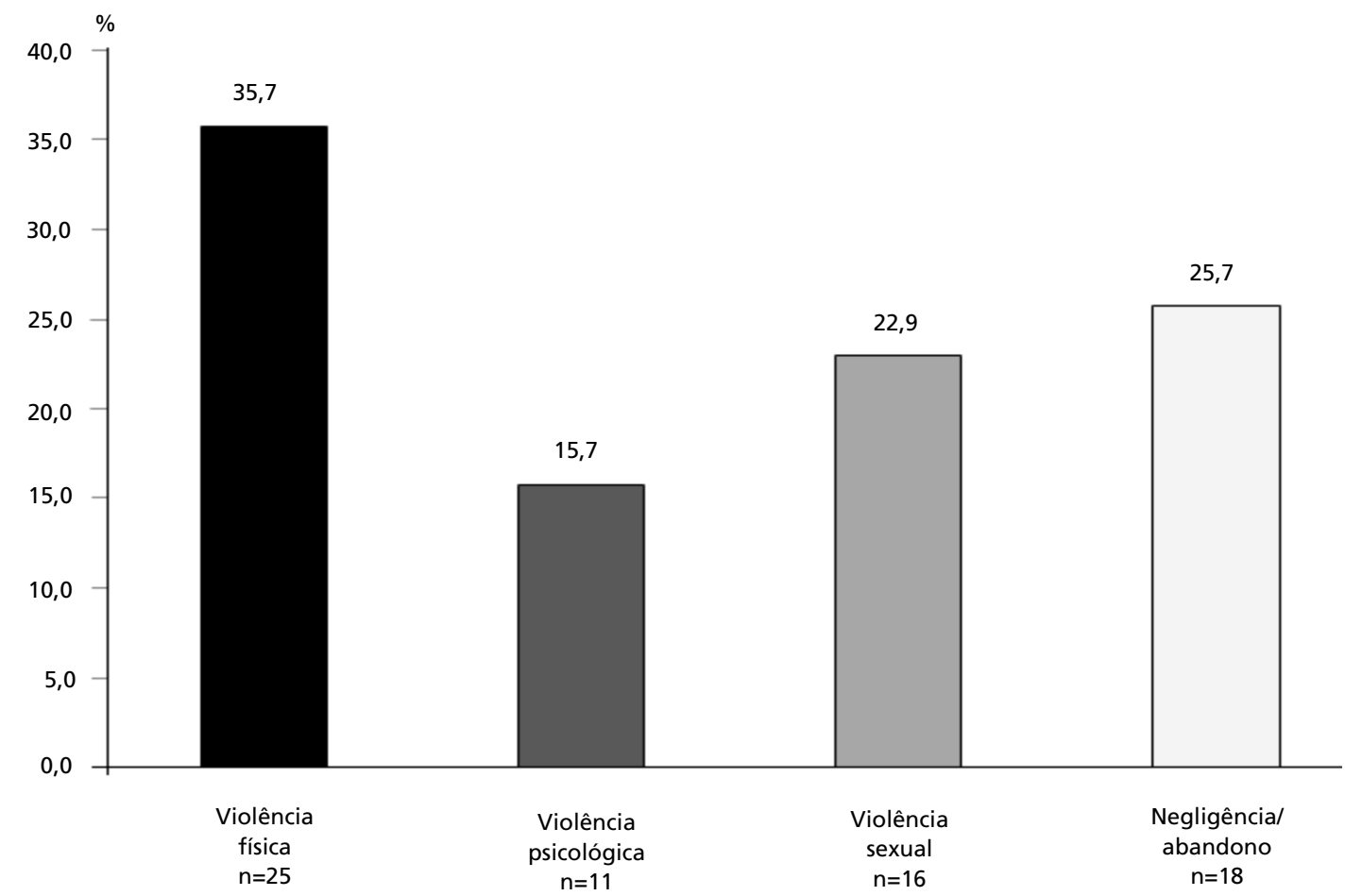


Relação entre perfil do profissional e atendimento de crianças e adolescentes suspeitos e/ou confirmados de sofrer violência intrafamiliar.

\begin{tabular}{|c|c|c|c|c|c|c|c|}
\hline & \multicolumn{6}{|c|}{ Atendimento de casos suspeitos e/ou confirmados de violência } & \multirow{3}{*}{$p^{*}$} \\
\hline & \multicolumn{2}{|c|}{ Sim } & \multicolumn{2}{|c|}{ Não } & \multicolumn{2}{|c|}{ Total } & \\
\hline & $\mathrm{n}$ & $\%$ & $\mathrm{n}$ & $\%$ & $\mathrm{n}$ & $\%$ & \\
\hline \multicolumn{8}{|l|}{ Tempo de formado (anos) } \\
\hline 1 a 5 & 12 & 30,8 & 15 & 30,0 & 27 & 30,3 & \\
\hline 6 a 10 & 8 & 20,5 & 7 & 14,0 & 15 & 16,9 & 0,679 \\
\hline Mais de 10 & 19 & 48,7 & 28 & 56,0 & 47 & 52,8 & \\
\hline Total & 39 & 100,0 & 50 & 100,0 & 89 & 100,0 & \\
\hline \multicolumn{8}{|l|}{ Área de atuação } \\
\hline Audiologia & 7 & 17,9 & 12 & 24,0 & 19 & 21,3 & \\
\hline Linguagem & 1 & 2,6 & 6 & 12,0 & 7 & 7,9 & \\
\hline Motricidade Orofacial & 2 & 5,1 & 3 & 6,0 & 5 & 5,6 & \\
\hline Voz & 1 & 2,6 & 2 & 4,0 & 3 & 3,4 & 0,542 \\
\hline Generalista & 8 & 20,5 & 7 & 14,0 & 15 & 16,9 & \\
\hline Mais de uma área de atuação & 20 & 51,3 & 20 & 40,0 & 40 & 44,9 & \\
\hline Total & 39 & 100,0 & 50 & 100,0 & 89 & 100,0 & \\
\hline \multicolumn{8}{|l|}{ Nível acadêmico } \\
\hline Graduação & 6 & 15,4 & 7 & 14,0 & 13 & 14,6 & \\
\hline Especialização/ Residência & 29 & 74,4 & 32 & 64,0 & 61 & 68,5 & 0,551 \\
\hline Mestrado & 4 & 10,3 & 9 & 18,0 & 13 & 14,6 & \\
\hline Doutorado & 0 & 0,0 & 2 & 4,0 & 2 & 2,2 & \\
\hline Total & 39 & 100,0 & 50 & 100,0 & 89 & 100,0 & \\
\hline \multicolumn{8}{|l|}{ Treinamento violência } \\
\hline Sim & 5 & 12,8 & 5 & 10,0 & 10 & 11,2 & \\
\hline Não & 34 & 87,2 & 79 & 90,0 & 79 & 88,8 & 0,286 \\
\hline Total & 39 & 100,0 & 50 & 100,0 & 89 & 100,0 & \\
\hline
\end{tabular}

*Teste exato de Fisher, exceto para tempo de formado (teste $\chi^{2}$ ).

Tabela 2

Distribuição de frequências de profissionais conforme condutas adotadas frente aos casos de violência.

\begin{tabular}{lrr}
\hline & $\mathbf{N}^{*}$ & $\%$ \\
\hline Encaminhou ao serviço social/psicologia & 24 & 34,3 \\
Aconselhou a família como proceder & 18 & 25,7 \\
Não tomou nenhuma conduta & 14 & 20,0 \\
Denunciou aos órgãos competentes & 2 & 2,9 \\
Encaminhou ao médico & 3 & 4,3 \\
Abandonou o caso & 0 & 0,0 \\
\hline
\end{tabular}

*Os profissionais poderiam responder mais de um item. 
Distribuição de frequência de fonoaudiólogos da rede pública de saúde sobre as instituições envolvidas na assistência à criança/adolescente vítima de violência. Recife, 2008.

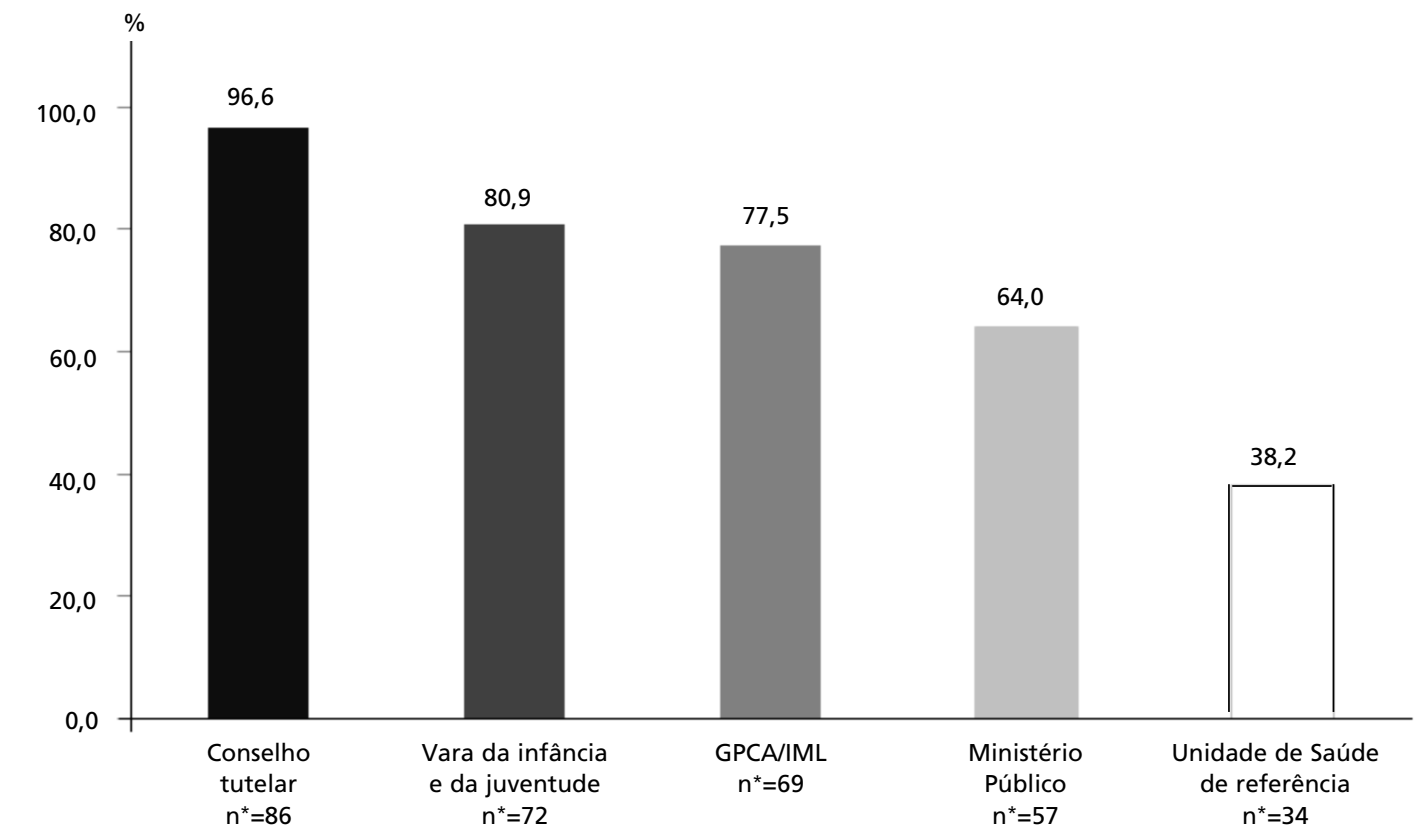

*Os profissionais poderiam responder a mais de um item; GPCA/IML= Gerência de Polícia da Criança e do Adolescente /Instituto de Medicina Legal.

\section{Discussão}

Os resultados obtidos neste estudo mostram que a violência intrafamiliar que acomete crianças e adolescentes está presente no cotidiano dos fonoaudiólogos. Semelhante aos resultados encontrados por Noguchi et al., 4 metade dos entrevistados do serviço público declarou ter atendido a crianças e/ou adolescentes vítimas de violência intrafamiliar.

Mais da metade dos fonoaudiólogos que identificaram casos de violência intrafamiliar eram generalistas ou trabalhavam em mais de uma área no campo da fonoaudiologia. Esse fato pode ser devido apenas ao maior contingente de indivíduos e não pela maior sensibilidade desses profissionais. Porém, sabe-se que a ampliação dos conhecimentos influencia positivamente não apenas na identificação das alterações fonoaudiológicas e seu tratamento, como também em aspectos que podem estar associados a sua práxis profissional, a exemplo dos casos da violência na infância e adolescência.18,19

O tempo de formado dos profissionais e o nível acadêmico não estiveram associados à identificação dos casos suspeitos de violência, embora os profissionais com especialização e residência foram os que atenderam ao maior número de casos. Esses resultados estão de acordo com os de outros estudos realizados no Brasil,4,14 porém torna-se oportuno lembrar que os achados podem ser devido a certas limitações do instrumento ao utilizar perguntas fechadas, as quais podem ter restringido as repostas dadas pelos profissionais, enquanto perguntas abertas poderiam possibilitar aos sujeitos expressarem outros aspectos não contemplados. Estudos que privilegiem a abordagem qualitativa, nesse caso, poderão ser úteis para ampliar a compreensão desses elementos.

Ao contrário do descrito na literatura, que tem reportado a influência positiva do acesso ao treinamento e à qualificação do profissional de saúde no aumento dos registros de casos de maus-tratos às instituições responsáveis 15,20,21 e, consequentemente, na redução da ocorrência do evento nos meses subsequentes, nesse estudo não se constatou associação entre treinamento ou curso que abordasse o tema violência e identificação dos casos de 
violência. Podem ter contribuído para tal resultado o tamanho relativamente restrito da amostra e o baixo contingente de profissionais capacitados.

Grande parte dos entrevistados declarou ter suspeitado de, pelo menos, um caso de violência, porém uma quantidade bastante reduzida denunciou os casos aos órgãos competentes. Essa conduta é distinta de outros profissionais da área de saúde, que, segundo alguns estudos, 14,22,23 tendem a notificar, com maior frequência, os casos de violência atendidos, mesmo diante da baixa ou nenhuma efetividade das medidas voltadas à solução do problema, que é expressa pela continuidade do ciclo de violência ou pelo abandono do tratamento da criança ou adolescentes, pelos pais ou responsáveis.

Os tipos de violência mais frequentemente identificados foram a violência física e a negligência/ abandono, resultados semelhantes aos observados nos atendimentos das áreas de enfermagem, medicina e odontologia. 16,24 Esse fato pode ser atribuído à sua fácil detecção, uma vez que esses tipos de violência deixam marcas visíveis ou retratam a falta de cuidados gerais com a criança, o que não ocorre nos outros tipos de violência.

O problema da subnotificação de casos de violência intrafamiliar pelos profissionais de saúde deve-se, segundo alguns autores,5,20,25,26 ao despreparo dos profissionais para o enfrentamento do problema, ocasionado pela pouca atenção dada pelas universidades, que não têm contemplado o assunto em sua grade curricular, e pela falta de capacitação e informação recebidas durante a prática profissional. Além disso, o código de ética profissional não deixa claras a responsabilidade e a atitude que deverão ser adotadas frente aos casos de violência, mencionando apenas dois itens que sugerem o tema: no capítulo $\mathrm{V}$, artigo nono, referente ao relacionamento, afirma que "o fonoaudiólogo deve respeitar o cliente e não permitir que ele seja desrespeitado", e, no capítulo VI, artigo 13, faz menção à necessidade de quebra do sigilo profissional nas "situações em que o seu silêncio ponha em risco a integridade do profissional, do cliente e da comunidade". No entanto, não apresenta a questão da obrigatoriedade da notificação da violência intrafamiliar. 27,28

$\mathrm{O}$ desconhecimento do profissional quanto à sua responsabilidade como cuidador da proteção de casos suspeitos e/ou confirmados de violência contra crianças e adolescentes pode ser um fator que comprometa a condução dos casos.

Utilizando como base o modelo biomédico, a prática do fonoaudiólogo tem-se caracterizado, durante muitos anos, pela sua forma de atuação isolada, com um olhar direcionado unicamente às alterações fonoaudiológicas e desarticulada dos outros profissionais da saúde. Esse fato pode ser constatado ao se analisar a história da fonoaudiologia, uma das mais jovens áreas da ciência da saúde, 29 pela forma fragmentada e centrada na produção de atos, sem considerar o paciente em sua integralidade. ${ }^{30}$ Essa situação, no entanto, vem-se modificando gradativamente no país como reflexo da maior inserção do fonoaudiólogo na rede pública de saúde, cujas práticas têm progressivamente se adequado aos princípios e diretrizes do SUS.

A principal conduta dos fonoaudiólogos frente aos casos de violência foi o encaminhamento para o serviço social/psicologia, existindo a pouca prática dos profissionais da notificação dos casos de maustratos infanto-juvenis, principal ação pontual contra a violência. 25

A maioria dos fonoaudiólogos mostrou ter bom conhecimento dos órgãos a serem acionados em caso de violência contra crianças e adolescentes, sendo o Conselho Tutelar a instituição mais citada para encaminhamento dos casos, corroborando os resultados de Noguchi. ${ }^{3}$

Em que pesem as limitações do desenho de estudo, que não permitem extrapolação dos seus resultados, acredita-se que situações semelhantes, possivelmente, ocorram em outras regiões do Brasil.

Os resultados alertam para a gravidade do problema da violência intrafamiliar contra crianças e adolescentes e mostraram o despreparo do fonoaudiólogo no enfrentamento do problema. Sendo assim, sugere-se a inclusão do tema na grade curricular dos cursos de graduação em fonoaudiologia, além da oferta de cursos de capacitações e de educação continuada aos profissionais que atuam nos serviços de saúde públicos e privados. Recomenda-se a realização de mais estudos, preferencialmente prospectivos e envolvendo maior número de profissionais para o melhor conhecimento do problema.

\section{Referências}

1. Gomes R, Junqueira MFPS, Silva CO, Junger WL. A abordagem dos maus-tratos contra a criança e o adolescente em uma unidade pública de saúde. Ciênc Saúde Coletiva. 2002; 7: $275-83$. 
2. Weber LND, Viezzer AP, Brandenburg OJ, Zocche CRE. Famílias que maltratam: uma tentativa de sociabilização pela violência. Psico USF. 2002; 7:163-73.

3. Lima PD, Farias MGM. Condutas adotadas pelos profissionais de saúde com crianças hospitalizadas vítimas de violência. Rev Eletrônica Enferm. 2008; 10: 643-53.

4. Noguchi MS, Assis SG, Santos NC. Entre quatro paredes: Atendimento fonoaudiológico à criança e adolescente vítima de violência. Ciênc Saúde Coletiva. 2004; 9: 963-73.

5. Silva MAI, Ferriani MGC. Violência domestica: do visível ao invisível. Rev Latino-am Enferm. 2007; 15: 275-81.

6. Noguchi MS, Assis SG. Fonoaudiologia e violência intrafamiliar contra criança: identificação de casos e prevenção. Pró-fono. 2003; 15: 199-206.

7. Deslandes, SF. O Atendimento às vítimas de violência na emergência: "prevenção numa hora dessas?". Ciênc Saúde Coletiva. 1999;4:81-94.

8. Silva LMP, Nascimento CAD, Silva IR, Guimarães KN, et al. Violência doméstica contra crianças e adolescentes. Brasília: Ministério da Saúde; 2002.

9. Sullivan PM, Brookhouser PE, Scanlan JM, Knutson JF, Schulte LE. Patterns of physical and sexual abuse of communicatively handicapped children. Ann Otol Rhinol Laryngol. 1991; 100: 188-94.

10. Deslandes SF Atenção a crianças e adolescentes vítimas de violência doméstica: análise de um serviço. Cad Saúde Pública. 1994; 10 (supl. 1): S177-87.

11. Allen R, Wasserman GA. Origins of language delay in abused infants. Child Abuse Negl. 1985; 9: 335-40.

12. Alves PM, Cavalcanti AL. Diagnóstico do abuso infantil no ambiente odontológico: uma revisão de literatura. Ciênc Biol Saúde Ponta Grossa. 2003; 9: 29-35.

13. Carvalho ACR, Barros SG, Alves AC, Gurgel CA. Maustratos: estudo através da perspectiva da delegacia de proteção à criança e ao adolescente em Salvador, Bahia. Ciênc Saúde Coletiva. 2009; 14: 539-46.

14. Pires JM, Goldani MZ, Vieira, EM, Nava TR, Feldens L, Castilhos K, Simas V, Franzon NS. Barreiras, para a notificação pelo pediatra, de maus-tratos infantis. Rev Bras Saúde Matern Infant. 2005; 5: 103-8.

15. Flaherty EG, Sege R. Barries to physician identification and reporting of abuse. Pediatr Ann. 2005; 34: 349-56.

16. Bankole OO, Denloye OO, Adeyemi, AT. Child abuse and dentistry: a study of knowledge and attitudes among Nigerian dentists. Afr J Med Sci. 2008; 37: 125-34.

17. Deslandes SF. Prevenir a violência: um desafio para profissionais de saúde. Rio de Janeiro: FIOCRUZ/ENSP/ CLAVES; 1994.
18. Silva N. Saúde pública e coletiva em pauta. Jornal do Conselho Federal de Fonoaudiologia (CFFA). 2005; janmar. (24): 05-06. Disponível em: http://www.fonoaudiologia.org.br/ publicacoes/ CFFa24.pdf

19. Andrade CRFA. Fases e níveis de prevenção em fonoaudiologia - ações coletivas e individuais. In: Vieira RM, Vieira MM, Avila CRB, Pereira LD. Fonoaudiologia e Saúde Pública; 2000. p. 81-103.

20. Starling SP, Heisler KH, Paulson JF, Youmans E. Child abuse training and knowledge: a national survey of emergency medicine, family medicine, and pediatric residents and program directors. Pediatrics. 2009; 12: 595-602.

21. Agirtan CA, Akar T, Akbas S, Akdur R, Aydin C, Aytar G, et al. Contributing Multidisciplinary Teams (MDT). Establishment of interdisciplinary child protection teams in Turkey 2002-2006: Identifying the strongest link can make a difference! Child Abuse Negl. 2009; 33: 247-55.

22. Martins CS, Ferriani MGC, Silva MAI, Zahr NR, Arone KMB, Roque EMST. A dinâmica familiar na visão de pais e filhos envolvidos na violência doméstica contra criança. Rev Latino-am Enferm. 2007; 15(5).

23. Silveira JLGC, Mayrink S, Neto OBS. Maus-tratos na infância e adolescência: casuística, conhecimento e prática dos cirurgiões dentistas. Pesq Bras Odontoped Clin Integr. 2005; 5: 19-126.

24. Lima PD, Farias GM. Condutas adotadas pelos profissionais de saúde com crianças hospitalizadas vítimas de violência. Rev Eletrônica Enferm. 2008; 10: 643-53.

25. Gonçalves HS, Ferreira AL. A notificação da violência intrafamiliar contra crianças e adolescentes profissionais de saúde. Cad Saúde Pública. 2002; 18: 315-9.

26. Melton GB. Mandated reporting: a policy without reason. Child Abuse Negl. 2005; 29: 9-18

27. Brasília. Conselho Federal de Fonoaudiologia. Código de Ética Profissional. 2004; mar. [acesso em 3 jan 2009] Disponível em: http://www.fonoaudiologia.com/informa/ etica

28. Saliba O, Garbin CAS, Garbin AJI, Dossi AP. Responsabilidade do profissional de saúde sobre a notificação de casos de violência doméstica. Rev Saúde Pública. 2007; 41: 472-7.

29. Befi D. A inserção da fonoaudiologia na atenção primaria à saúde. In: Fonoaudiologia na atenção primaria à saúde. São Paulo: Lovise; 1997. p. 25-33.

30. Machado MFAS, Monteiro EMLM, Queiroz DT, Vieira, NFC, Barroso, MGT. Integralidade, formação de saúde e as propostas do SUS - uma revisão conceitual. Ciênc Saúde Coletiva. 2007; 12: 335-42.

Recebido em 14 de setembro de 2010

Versão final apresentada em 29 de dezembro de 2010

Aprovado em 11 de janeiro de 2011 